\title{
Resonance Modes at Harmonics Frequencies in Electrical Networks
}

\author{
Lidiia I. Kovernikova ${ }^{1, *}$ \\ ${ }^{1}$ Melentiev Energy Systems Institute SB RAS, Irkutsk, Russian Federation
}

\begin{abstract}
Resonance modes at harmonic frequencies in electrical networks are a serious problem. They arise due to the availability of electrical equipment with capacitive and inductive elements. The values of the harmonics of currents and voltages increase at resonances. The voltage quality indices in resonant modes exceed the limit values. Harmonics cause energy losses in electrical equipment, reduce its service life, create economic damage. Capacitor banks are often damaged by resonances. Network nodes with resonant circuits and resonant harmonics can be determined using the frequency characteristics of the nodal reactance (susceptance). The paper presents an algorithm and HARMONICS software for the analysis and forecasting of resonance modes, the results of studies of resonance modes in the high-voltage networks of Eastern Siberia.
\end{abstract}

\section{Introduction}

Resonance modes at harmonics frequencies in power grids have been [1-5] and remain a big challenge [6-10]. They occur due to availability of equipment with capacitance and reactive elements $[11,12]$. The hazard of resonance modes lies in the fact that if a non-sinusoidal current with harmonic components multiple to the main frequency (harmonics) and/or non-multiple to the main frequency (interharmonics) on which the resonance circuit occurred, runs in the grid, then current and voltage surge may occur at those harmonics. Current and voltage harmonics cause additional power losses in the electric equipment; they damage it, reduce its service life and thus cause economic losses. To avoid negative consequences of resonance modes, standardization documents provide for the occurrence of resonances.

"Guidelines for monitoring and analyzing the power quality in public power supply system" [4] that were in force in Russia in 2002-2008 stated that "non-sinusoidal nature of voltage in the point of common connection (PCC) may be caused both by distorting utilities of consumers connected directly to PCC and by equipment of power supply companies operating in the modes favoring the occurrence of non-sinusoidal nature of their volt-ampere characteristics or occurrence of resonance modes". In 2003 in the Project Technological rules for the wholesale electricity market [5] it was noted that "network companies during their operation are responsible for network modes unfavorable or hazardous for equipment and consumers (resonance modes...)". In 2019 a document "Unified technical policy in the power grid complex of Rosseti" [13] mentioned that "Usage of a capacitor is allowed subject to avoidance of resonance phenomena in all the operating conditions of a network". It was also noted that "when selecting the power factor correction unit (PFCU) containing capacitor banks (CB) for the network section where frequent distortions of current and voltage curves occur, PFCU shall be checked (CB in particular) for possible overloading by harmonic currents".

The paper offers an algorithm for determination of resonance nodes, harmonics and interharmonics on the base of analysis of the results of calculations made using HARMONICS software. HARMONICS software was developed in ESI SB RAS [14]. The analysis allows identification of network sections and harmonics with the presence of resonance modes or modes close to them. The paper presents the results of studies on the resonance modes in the high-voltage networks in the East Siberia. An algorithm and HARMONICS software can be used for forecasting the resonance modes for power quality control in the course of networks operation.

\section{An algorithm for analysis of resonance modes}

Theoretical foundations of electric power engineering [11] state that two types of resonance modes may occur in the electrical circuit: series resonance, i.e., voltage resonance, and parallel resonance, i.e., current resonance.

Voltage resonance occurs in case of series connection of resistance, inductive reactance and capacitive reactance. Circuit reactance at resonance equals zero. Circuit impedance is active and reaches its lowest value. Current and voltage at the circuit entry match in phase. Current has the highest value. If value of inductive and capacitive reactances exceeds that of resistance, then value of voltage on the capacitive and inductive reactances will exceed voltage at the circuit entry.

\footnotetext{
* Corresponding author: kovernikova@isem.irk.ru
} 
Current resonance occurs at parallel connection of resistance, inductive and capacitive reactances. Circuit susceptance at resonance equals zero. Circuit admittance reaches its minimum value. Current and voltage in the unbranched part of the circuit match in phase. The value of current happens to be the lowest. Values of currents in parallel branches with inductive and capacitive reactances may exceed current in unbranched part of the circuit.

For analysis of the network modes at harmonic frequencies the electric network is represented by a current source and admittance relative to any node. Modes are calculated by solving the system of equations

$$
U_{h}=Z_{h} I_{h},
$$

where $\boldsymbol{h}$ - harmonic order; $\boldsymbol{U}_{\boldsymbol{h}}$ - column matrix of nodal voltages of the $h$-th harmonic that are to be determined; $\boldsymbol{I}_{\boldsymbol{h}}$ - column matrix of nodal currents of the $h$-th harmonic that are generated by non-linear loads; $\boldsymbol{Z}_{\boldsymbol{h}}$ matrix of self- and mutual impedances of the network nodes, which is obtained as result of inversion of the nodal admittance matrix $\boldsymbol{Y}_{\boldsymbol{h}}$ [15].

Data of the system of equations (1) for analysis of resonance conditions in any node are used for computation of the following parameters: $K_{U(h)}$ - the $h$ th harmonic factor; $I_{h}-$ current value of the $h$-th harmonic; $y_{h}=1 / z_{h}=1 / \sqrt{r_{h}^{2}+x_{h}^{2}}$ - admittance of the network node; $g_{h}=r_{h} / \sqrt{r_{h}^{2}+x_{h}^{2}}-$ conductance of the network node; $b_{h}=x_{h} / \sqrt{r_{h}^{2}+x_{h}^{2}}$ - susceptance of the network node. $K_{U(h) 95 \%}, K_{U(h) 100 \%}$ are limit values of the index $K_{U(h)}$ used in the analysis for $95 \%$ and $100 \%$ of measurement time specified in [16]. Resonance harmonics can be adjusted by computing the frequency characteristics for $y_{h}, g_{h}$, and $b_{h}$. For a largedimensionality network it is a sophisticated problem that requires account of a large number of network elements, significant time for calculating and analysis of frequency characteristics. Experience of studying the harmonics conditions allowed development of an algorithm for searching the resonance conditions on the base of the results of calculations using HARMONICS software that facilitates determination of nodes and harmonics with resonance modes. The modes are initially calculated for $3,5,7,9,11,13,17,19,23$, and 25-th harmonics. Measured values of indexes $K_{U(h)}$ on the listed harmonics in the electric networks, as a rule, exceed the limit values specified in [16]. For adjusting the resonance harmonics the range of harmonics is then changed.

An algorithm for determination of resonance nodes and harmonics includes provisions listed further.

1) Computation of mode parameters and nodal parameters on harmonics $3,5,7,9,11,13,17,19,23,25$ for determination of values $K_{U(h)}, I_{h}, y_{h}, g_{h}$, and $b_{h}$.
2) Analysis of parameters as per Item 1) for the nodes where values $K_{U(h)}$ exceed the limit values $K_{U(h) 95 \% \text {, }}$

$K_{U(h) 100 \%}$.

In the course of the analysis it should be kept in mind that index $K_{U(h)}$ exceeds the limit value not only in the resonance mode but at high value of current $I_{h}$ in the node as well.

Since susceptance in the resonance mode equals zero, conductance and admittance are equal in value, then combinations of these values are searched for determination of nodes with resonance on the computed harmonics.

Nodes where modes are close to resonance conditions are also identified. Numerical values of conductance and admittance are rather close, whereas the value of susceptance is much lower than conductance and admittance.

For identification of resonance modes or modes close to them the signs of susceptance on the adjacent harmonics are analyzed. Change of the sign evidences availability of the resonance mode on the interval of harmonics. Susceptance sign of the node may change several times, which evidences availability of the resonance modes on several harmonics simultaneously.

As a result of Item 2) the nodes, harmonics or harmonics intervals are determined where resonance modes or modes close to them occur.

3) Analysis of parameters as per Item 1) for the nodes where values $K_{U(h)}$ ) do not exceed the limit values $K_{U(h) 95 \%}, K_{U(h) 100 \%}$.

For finding the resonance modes, the values of admittances are analyzed for searching the nodes and harmonics with low values of admittances. Susceptances are further analyzed in the nodes with low values of admittances for identification of the sign change.

As a result of Item 3) the nodes, harmonics or harmonic intervals are determined where resonance modes or modes close to them occur.

4) Determination of the number of the resonance harmonic or interharmonic on the interval of harmonics obtained in Items 2) and 3).

For identification of the number of a resonance harmonic the frequency characteristic of susceptance is computed from $50 \mathrm{~Hz}$ on the harmonics interval at a step of $0.5 \%$. The frequency characteristic curve is constructed. The harmonic or interharmonic number at which the susceptance sign changes, i.e., resonance occurs, is determined using frequency characteristic.

For adjusting the number of a resonance harmonic the harmonic interval is reduced with account of the results of above calculations, and frequency characteristic is computed anew from the presumed resonance harmonic at a step of $0.2 \%$. The obtained frequency characteristic of susceptance is used for adjusting the number of a resonance harmonic. If necessary, calculations are repeated at a lesser step.

5) Determination of resonance harmonics in the node at different changes in the network. Connection and disconnection of transmission lines, capacitors, passive 
filters, and changing loads can lead to resonant modes. For assessing of occurrence of resonance modes in the node in case of changes in the network, Items 1) - 4) of the algorithm shall be fulfilled.

\section{Results of the analysis of resonance modes in the networks of East Siberia}

\subsection{An example of using the algorithm for searching the resonance modes in one of the nodes of the calculated scheme}

Analysis of the mode parameters when searching for the resonance modes in Node 2401 of the calculated network whose fragment is shown in Fig. 1 is given as an example.

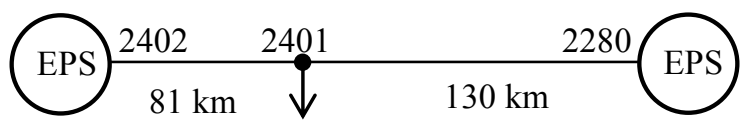

Fig. 1. Fragment of the calculated network with Node 2401.

Parameters as per Item 1) for the analyzed node are given in Table 1 and Table 2.

Table 1. Mode parameters of harmonics 3, 5, 7, 9, 11

\begin{tabular}{|l|c|c|c|c|c|}
\hline \multicolumn{1}{|c|}{ Harmonic } & 3 & 5 & 7 & 9 & 11 \\
\hline$I_{h}, \mathrm{~A}$ & 21.35 & 10.70 & 7.30 & 2.78 & 7.39 \\
\hline$y_{h}$, p.u. & 5.02 & 2.15 & 1.01 & 0.92 & 1.39 \\
\hline$g_{h}$, p.u. & 0.69 & 0.37 & 0.76 & 0.76 & 1.22 \\
\hline$b_{h}$, p.u. & -4.97 & -2.11 & -0.67 & -0.52 & 0.67 \\
\hline$K_{U(h)}, \%$ & 0.98 & 1.15 & $\mathbf{1 . 6 7}$ & $\mathbf{0 . 6 9}$ & $\mathbf{1 . 2 2}$ \\
\hline$K_{U(h) 95 \%} \%$ & 1.5 & 1.5 & 1.0 & 0.4 & 1.0 \\
\hline$K_{U(h) 100 \%}, \%$ & 2.25 & 2.25 & 1.5 & 0.6 & 1.5 \\
\hline
\end{tabular}

Table 2. Mode parameters of harmonics 13, 17, 19, 23, 25

\begin{tabular}{|l|c|c|c|c|c|}
\hline \multicolumn{1}{|c|}{ Harmonic } & 13 & 17 & 19 & 23 & 25 \\
\hline$I_{h}, \mathrm{~A}$ & 9.70 & 2.48 & 4.61 & 2.91 & 0.78 \\
\hline$y_{h}$, p.u. & 1.29 & 3.19 & 4.76 & 2.88 & 1.4 \\
\hline$g_{h}$, p.u. & 1.29 & 2.16 & 4.75 & 2.27 & 1.16 \\
\hline$b_{h}$, p.u. & -0.04 & 2.34 & -0.16 & -1.76 & -0.78 \\
\hline$K_{U(h)}, \%$ & $\mathbf{1 . 7 2}$ & 0.18 & 0.22 & 0.23 & 0.13 \\
\hline$K_{U(h) 95 \%}, \%$ & 0.7 & 0.5 & 0.4 & 0.4 & 0.4 \\
\hline$K_{U(h) 100 \%}, \%$ & 1.05 & 0.75 & 0.6 & 0.6 & 0.6 \\
\hline
\end{tabular}

Index $K_{U(h)}$ exceed the limit values $K_{U(h) 95 \%}$ on the 7, 9, 11 and 13-th harmonics, and $K_{U(h) 10 \%}$ on the 7, 9 and 13-th harmonics (in bold).
Conductance and susceptance on the 7-th harmonic are close in value. Index $K_{U(7)}$ is high due to higher value of current on the 7-th harmonic. The value of all the conductances on the 9-th harmonic is of the same order, which evidences the lack of resonance in the vicinity of the 9-th harmonic. Susceptance on the interval of harmonics 9-11 changes from "minus" to "plus", which evidences the resonance of voltages. Admittance and conductance on the 11-th harmonic are close in value, whereas susceptance is half the admittance and conductance. So far as the value of susceptance is of the same order as admittance, we can assume that there is no resonance in the vicinity of the 11-th harmonic, and $K_{U(11)}$ exceeds the limit value due to high value of current on the 11-th harmonic. For adjusting the number of a resonance harmonic the frequency characteristics of susceptance shall be used. On the interval of harmonics 11-13 the susceptance sign changes from "plus" to "minus". Admittance and conductance on the 13-th harmonic are close in value, whereas susceptance is rather low as it has no impact on admittance. Thus, current resonance occurs on the interval of harmonics 11-13. For adjusting the number of resonance harmonic the frequency characteristic of susceptance shall be used. Susceptance sign on the interval of harmonics 13-17 changes from "minus" to "plus", which evidences the presence of the resonance of voltages. Values of all the conductances on the 17-th harmonic are of the same order. Comparison of the values of susceptances on the 13-th and 17-th harmonics evidences the voltage resonance on the harmonic close to the 13-th harmonic. Susceptance on the interval of harmonics 17-19 changes from "plus" to "minus", which evidences the resonance of currents. Admittance and conductance on the 19-th harmonic are close in value, whereas susceptance is rather low. We can conclude that current resonance occurred on the harmonic close to the 19-th harmonic.

Frequency characteristics of admittance, conductance and susceptance were computed on the harmonics intervals 3-25 using HARMONICS software. From Fig. 2 it follows that frequency characteristics of susceptance intersects abscissa four times changing the sign: between 9-th and 10-th harmonics; two times between 12-th and 13-th harmonics and between 18-th and 19-th harmonics, which evidences the occurrence of four resonances. Results of the analysis of frequency characteristics of susceptance are given in Table 3. They correspond to activated state of lines in Fig. 1.

Disturbances that may cause change in the number of resonances and resonance harmonics in Node 2401 include disconnections of transmission lines connected to Node 2401. Frequency characteristics of susceptances of Node 2401 of transmission lines in Fig. 3 are given for comparison. It is obvious that the number of resonances and numbers of resonance harmonics for each state of a transmission line are different. 


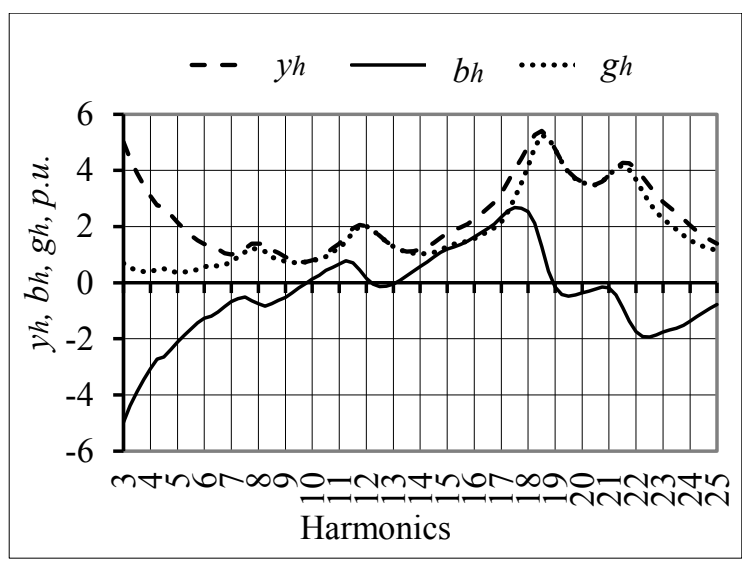

Fig. 2. Frequency characteristics of admittance, conductance and susceptance on the harmonics intervals 3-25.

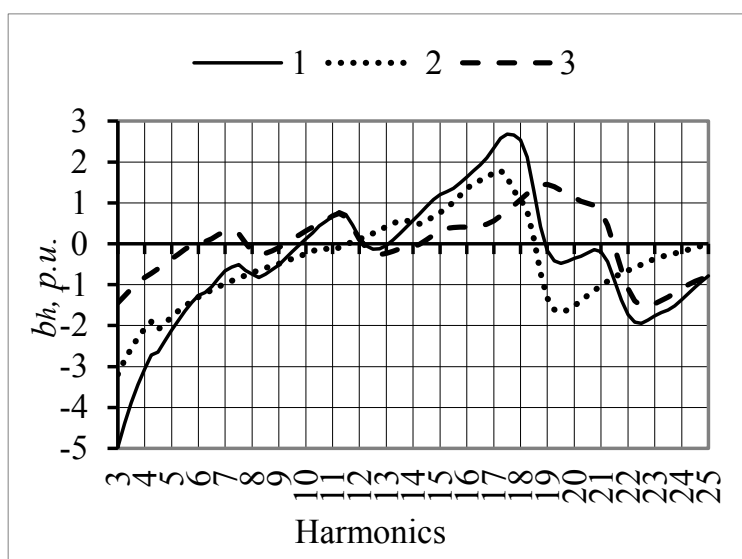

Fig. 3. Frequency characteristics of susceptance of Node 2401: 1 - two lines are turned on; 2 - line 2401-2402 is turned on, line 2401-2280 is turned off; 3 - line 2401-2402 is turned off, line 2401-2280 is turned on.

Table 3 gives intervals of harmonics where there is a resonance harmonic and the values of susceptances corresponding to the interval of resonance harmonics for three states of transmission lines.

Table 3. Results of analysis of frequency characteristics of susceptance

\begin{tabular}{|c|c|c|}
\hline $\begin{array}{l}\text { State of } \\
\text { transmission } \\
\text { lines }\end{array}$ & $\begin{array}{c}\text { Interval } \\
\text { resonant } \\
\text { harmonics and } \\
\text { interharmonic }\end{array}$ & $\begin{array}{c}\text { Interval } \\
\text { values } \\
b_{h}, \text { p.u. }\end{array}$ \\
\hline \multirow{4}{*}{$\begin{array}{l}\text { Both lines } \\
\text { are turned on }\end{array}$} & $9.75-10.00$ & $(-0.03)-(0.13)$ \\
\hline & $12.00-12.25$ & $(0.16)-(-0.05)$ \\
\hline & $13.00-13.25$ & $(-0.04)-(0.09)$ \\
\hline & $18.75-19.00$ & $(0.42)-(-0.16)$ \\
\hline \multirow{2}{*}{$\begin{array}{l}2401-2402 \text { is } \\
\text { turned on, } \\
2401-2280 \text { is } \\
\text { turned off }\end{array}$} & $11.50-11.75$ & $(-0.02)-(0.05)$ \\
\hline & $18.50-18.75$ & $(0.12)-(-0.72)$ \\
\hline \multirow{6}{*}{$\begin{array}{l}2401-2402 \text { is } \\
\text { turned off, } \\
2401-2280 \text { is } \\
\text { turned on }\end{array}$} & $5.75-6.00$ & $(-0.03)-0.06$ \\
\hline & $7.75-8.00$ & $(0.02)-(-0.18)$ \\
\hline & $9.00-9.25$ & $(-0.10)-(0.04)$ \\
\hline & $12.00-12.25$ & $(0.11)-(-0.10)$ \\
\hline & $14.00-14.25$ & $(-0.01)-(0.10)$ \\
\hline & $21.00-21.25$ & $(0.44)-(-0.07)$ \\
\hline
\end{tabular}

\subsection{Impact of a capacitor bank on occurrence of resonance conditions}

Fig. 4 shows a fragment of the calculated network where a 50 MVAr capacitor bank owned by a power supply company is connected to Node 1980. Capacitor bank is activated for maintaining the voltage at the main frequency in conformity with requirements of standardizing documents. A traction substation is connected to Node 1981. Values $K_{U(3)}$ and $K_{U(5)}$ measured in three phases of Node 1981 before and after activating the capacitor bank are given in Figs. 5 and 6 . Capacitor bank was activated after the 19-th minute. It is obvious those after activating the capacitor bank the values $K_{U(3)}$ and $K_{U(5)}$ increased. They exceeded the limit values specified in [16].

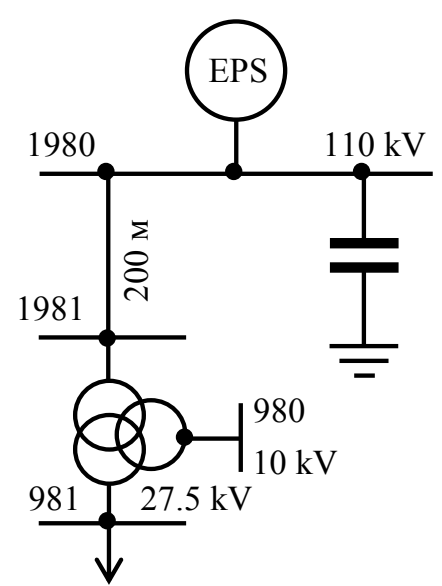

Fig. 4. Fragment of the calculated network with capacitor bank.

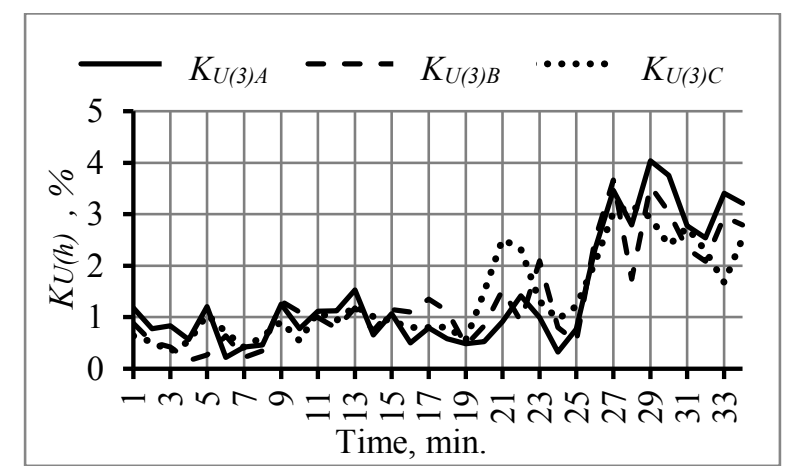

Fig. 5. $K_{U(3)}$ measured in three phases.

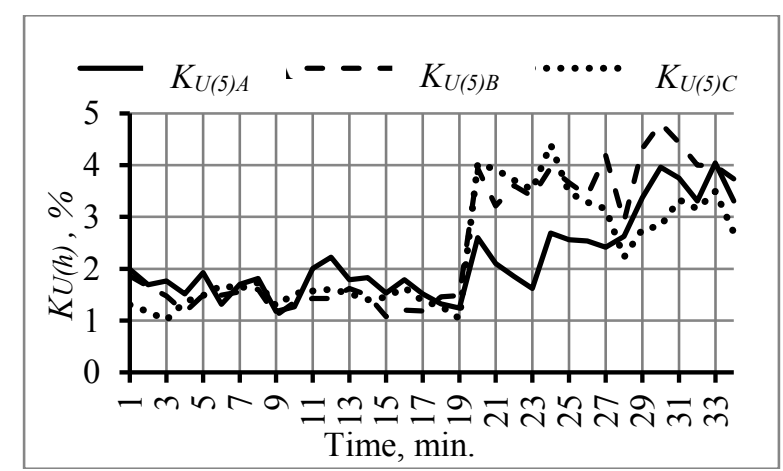

Fig. 6. $K_{U(5)}$ measured in three phases. 
Values $K_{U(h)}$ for harmonics 7, 9, 11, 13, 17, 19, 23 and 25 are given in Figs. 7 and 8. After activating the capacitor bank the values of all the listed harmonics decreased. Their values are less than half of the limit ones.

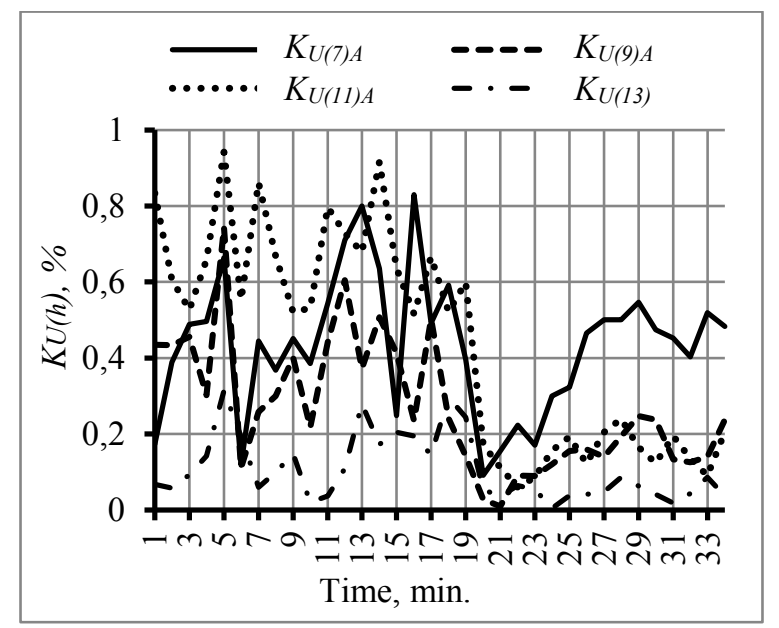

Fig. 7. $K_{U(7)}, K_{U(9)}, K_{U(11)}, K_{U(13)}$ measured in phase A.

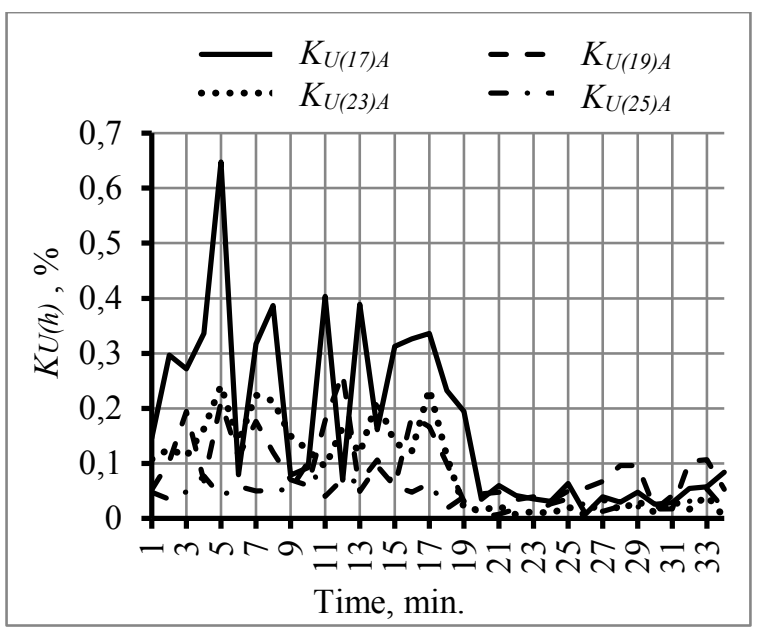

Fig. 8. $K_{U(17)}, K_{U(19)}, K_{U(23)}, K_{U(25)}$ measured in phase A.

For analysis of the impact of capacitor bank on the network mode at the harmonics frequencies, mode parameters in Node 1981 without a capacitor bank were calculated using HARMONICS software, and they were calculated after the bank activation. Results of calculations are given in Table 4.

Table 4. Parameters of the node and mode

\begin{tabular}{|l|c|c|}
\hline \multirow{2}{*}{ Parameter } & \multicolumn{2}{|c|}{ Harmonic } \\
\cline { 2 - 3 } & 3 & 5 \\
\hline$g_{h N}, S$ & 0.0046 & 0.0092 \\
\hline$g_{h C B}, S$ & 0.0 & 0.0 \\
\hline$g_{h N+C B}, S$ & 0.0046 & 0.0092 \\
\hline$b_{h N}, S$ & -0.0116 & -0.0125 \\
\hline$b_{h C B}, \mathrm{~S}$ & 0.0076 & 0.0127 \\
\hline$b_{h N+C B}, \mathrm{~S}$ & -0.0039 & 0.0003 \\
\hline$y_{h+C B}, S$ & 0.0060 & 0.0092 \\
\hline$K_{U(h)}, \%$ & 1.14 & $\mathbf{1 . 7 5}$ \\
\hline$K_{U(h) C B}, \%$ & $\mathbf{2 . 3 6}$ & $\mathbf{2 . 9 6}$ \\
\hline
\end{tabular}

Notations in the Table: $g_{h C B}$ - conductance of a capacitor bank; $g_{h N}$ - conductance of a node; $g_{h N+C B}-$ a sum of conductance of capacitor bank and of node; $b_{h C B}-$ susceptance of capacitor bank; $b_{h N}$ - susceptance of node; $b_{h N+C B}$ - a sum of susceptance of capacitor bank and susceptance of node; $y_{h N+C B}$ - admittance of a capacitor bank and node; $K_{U(h)}$ - value of the index before activation of a capacitor bank; $K_{U(h) C B}$ - value of the index after activation of a capacitor bank. Data in the table demonstrate that susceptance of the node before activation of a capacitor bank $\left(b_{h N}\right)$ had a "minus" sign on the 3-rd and 5-th harmonics. After activation of a capacitor bank the susceptance $\left(b_{h N+C B}\right)$ of the 5-th harmonics changed the sign to "plus". Change of susceptance sign evidences the voltage resonance on the interval of harmonics from 3-rd to 5-th. As a result, the values $K_{U(3)}$ and $\left.K_{U(5)}\right)$ increased and exceeded the limit values specified in [16]. .

\subsection{Resonance modes in the $220 \mathrm{kV}$ network}

Fig. 9 shows a fragment of the calculated $220 \mathrm{kV}$ network from Node 2012 to Node 2880. The distance between nodes is about $840 \mathrm{~km}$. The $220 \mathrm{kV}$ network powers more than 20 traction substations.

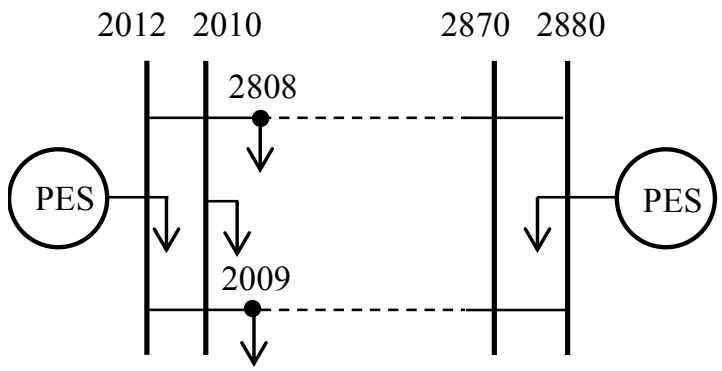

Fig. 9. Fragment of the calculated $220 \mathrm{kV}$.

Parameters of modes and nodes of the network for 3, 5, 7 and 11-th harmonics were calculated using HARMONICS software. Results of calculations are presented in the form of graphs in Figs. 10-13.

Fig. 10 shows computed parameters for the 3-rd harmonic.

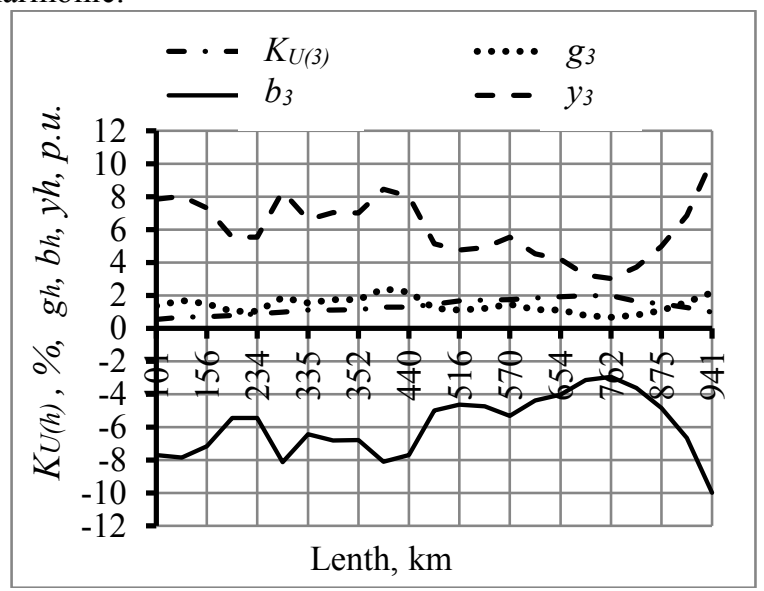

Fig. 10. Parameters of nodes and mode on the 3-rd harmonic. 
The susceptance curve does not intersect the abscissa, which means that there are no resonances on the 3-rd harmonic. The value $K_{U(3)}$ increases with decrease of admittance.

On the 5-th harmonics (Fig. 11) the resonances occur at a distance of $400-700 \mathrm{~km}$ from Node 2012. Susceptance changes its sign four times. Two serial and two parallel resonances occur in the network. The value of susceptance in the neighbourhood of resonance harmonics reduces; values of admittance and conductance are also reduced and become close in value. Index $K_{U(5)}$ increases. In the vicinity of resonances it reaches its maximum that exceeds the limit value $K_{U(5) 95 \%}$.

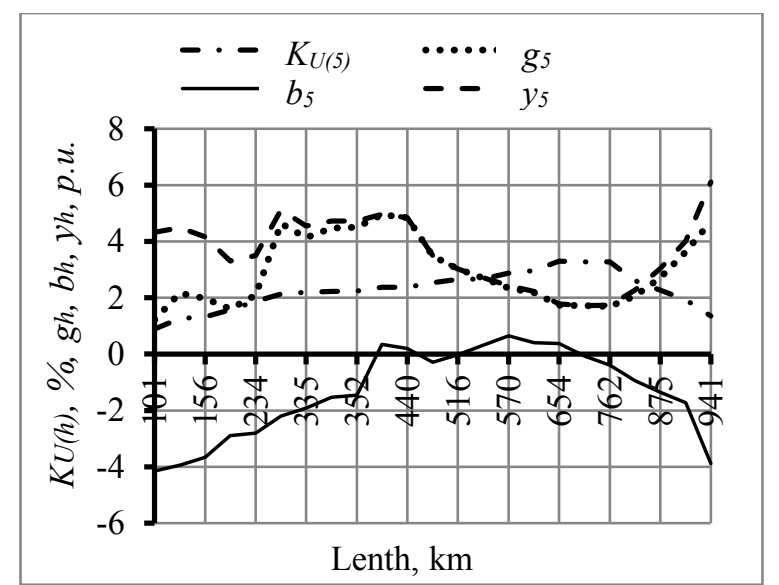

Fig. 11. Parameters of nodes and mode on the 5-th harmonic.

Resonance circuits on the 7-th harmonics were formed from the point of $234 \mathrm{~km}$ to $941 \mathrm{~km} \mathrm{(Fig.} \mathrm{12).}$ Susceptance changes its sign four times. Two voltage resonances and two current resonances occurred in the network. The values of $K_{U(7)}$ exceeded $K_{U(7) 95 \%}$ but it turned out to be lower than the limit value $K_{U(7) 100 \%}$.

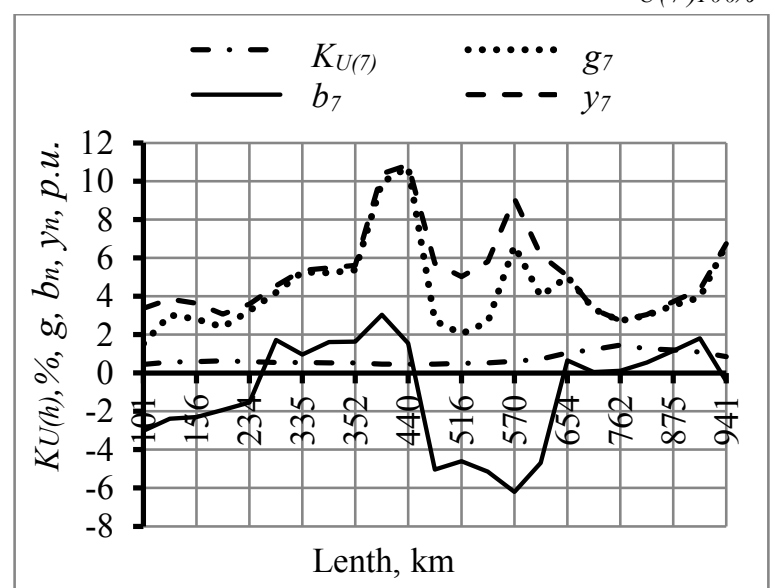

Fig. 12. Parameters of nodes and mode on the 7-th harmonic.

Resonance circuits on the 11-th harmonic were formed throughout the network. Susceptance changes the sign nine times. Nine resonance circuits occurred in the network, five of which correspond to voltage resonance and four correspond to current resonance. Since currents of the 11-th harmonic of a traction station are negligible, index $K_{U(11)}$ ) does not exceed the limit value of $K_{U(11) 10 \%}$.

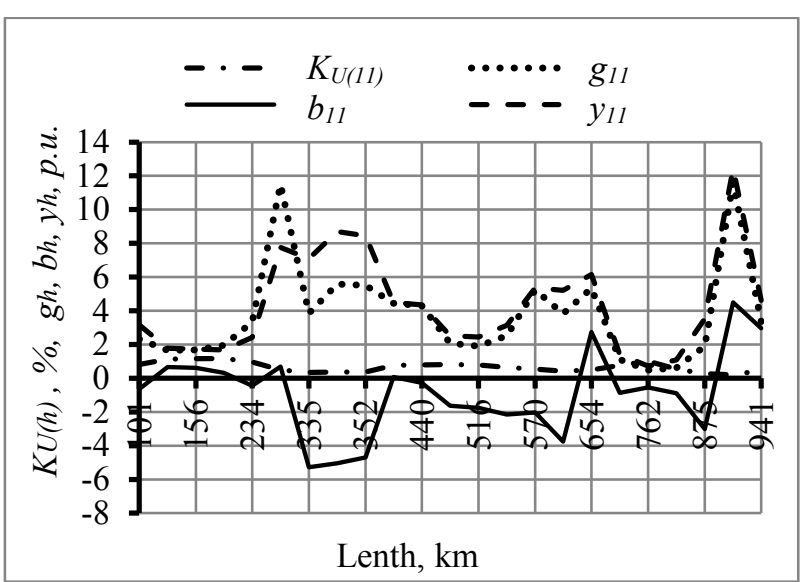

Fig. 13. Parameters of nodes and mode on the 11-th harmonic.

\section{Conclusions}

1. Resonance modes in the network nodes are of random nature, cover vast areas of the network and are caused by different changes in the network, very often at several harmonics and interharmonics simultaneously, and are among the causes why $K_{U(h)}$ exceeds the limit values specified in State Standard 32144-2013.

2. Tables generated using HARMONICS software reveal the mechanism of voltage harmonics occurrence in the network nodes and together with frequency characteristics of conductance, susceptance and admittance identify resonance modes.

3. The algorithm proposed for analysis of resonance conditions at the harmonics frequencies together with HARMONICS software can be applied for power quality control during networks operation for the purpose of forecasting the resonance conditions.

The research is conducted in the framework of the research project №AAAA-A17-117030310438-1 of the program of fundamental research of Siberia Branch of the Russian Academy of Sciences III.17.4.

\section{References}

1. K. Geddey, D.C. Smith, P.O.Wright, P.M. Hart, G.E. Littler, Control of harmonic distortion on Australian Electricity supply networks. CIGRE, 1986, in the book "The impact of high voltage electrical installations on the environment". Moscow, Energoatomizdat, (1988)

2. K. Hlava, I. Kabrhel, J. Smid, M. Blasko, Experimental investigation of harmonic impedances and other electrical parameters of high voltage network. CIGRE, 1986, in the book "The impact of high voltage electrical installations on the environment". Moscow, Energoatomizdat, (1988) 
3. Tutorial harmonics modeling and simulation. IEEE power engineering society. IEEE Catalog number: 98TR125-0.

4. RD 153-34.0-15.501-00. Guidelines for monitoring and analyzing the power quality in public power supply systems. Analysis of the power quality. Moscow, (2002)

5. Technological rules for the wholesale electricity market. Project. Moscow, (2003)

6. T. Vinnal, T. Sakkos, K. Janson, M. Jarkovoi, Resonances in LV industrial networks when using shunt capacitors for power factor correction, Proceedings of 23th International Conference on Electricity Distribution - CIRED 2015, Lyon, 15-18 June (2015)

7. J. Meyer, R. Stiegler, P. Schegner, I. Röder, A. Belger, Harmoic resonances in residential low voltage networks caused by consumer electronics, Proceedings of $24^{\text {th }}$ International Conference on Electricity Distribution - CIRED 2017, Glasgow, 12-15 June (2017)

8. L. Eggenschwiler, O. Galland, D. Chollet, F. Decorvet, D. Roggo, P. Favre-Perrod, Frequency scans and resonance mode analysis for resonance problems identification in power networks in presence of harmonic pollution, Proceedings of 24th International Conference on Electricity Distribution - CIRED 2017, Glasgow, 12-15 June (2017)

9. B. Mohamadi Kalesar, J. Behkesh Noshahr, Capacitor bank behavior of cement factory in presence of supraharmonics resulted from switching full power frequency converter of generator (PMSG), Proceedings of 24th International Conference on Electricity Distribution - CIRED 2017, Glasgow, 12-15 June (2017)
10. A. Bottenberg, C. Debruyne, B. Peterson, J. Rens, J. Knockaert, J. Desmet, Network resonance detection using harmonic active power, Proceedings of 18th International Conference on Harmonics and Quality of Power (ICHQP), Ljubljana, Slovenia, May 13-16 (2018)

11. L.A. Neiman, K.S. Demirchan, Theoretical foundations of electrical engineering: In 2 Vol. Textbook for universities. Volume 1. - 3rd ed., revised. and add. - L.: Energoatomizdat. Leningr. Department, (1981)

12. G.J. Wakileh, Power system harmonics: fundamentals, analysis and filter design. Berlin; Heidelberg; New York; Barcelona; Paris: Springer, (2001)

13. Unified technical policy in the power grid complex of "Rosseti". Project. Moscow, (2019)

14. L.I. Kovernikova, HARMONICS software for analysis and normalization of harmonic modes on $H V$ networks, Proceedings of the International Scientific and Practical Conference, Moscow, 23-25 November (2016)

15. L.A. Zhukov, I.P. Stratan, Steady-state modes of complex electrical networks and power systems: calculation methods, Energiya, (1979)

16. State Standard 32144-2013. Electric energy. Electromagnetic compatibility of technical equipment. Power quality requirements in public electricity supply systems. Moscow, Standartinform, (2014) 